Published in final edited form as:

Future Microbiol. 2013 March ; 8(3): 353-365. doi:10.2217/fmb.12.150.

\title{
Stress responses in the opportunistic pathogen Acinetobacter baumannii
}

\author{
Steven E Fiester ${ }^{1}$ and Luis A Actis ${ }^{1, *}$ \\ ${ }^{1}$ Department of Microbiology, Miami University, Oxford, $\mathrm{OH}$ 45056, USA
}

Abstract

Acinetobacter baumannii causes a wide range of severe infections among compromised and injured patients worldwide. The relevance of these infections are, in part, due to the ability of this pathogen to sense and react to environmental and host stress signals, allowing it to persist and disseminate in medical settings and the human host. This review summarizes current knowledge on the roles that environmental and cellular stressors play in the ability of $A$. baumannii to resist nutrient deprivation, oxidative and nitrosative injury, and even the presence of the commonly used antiseptic ethanol, which could serve as a nutrient- and virulence-enhancing signal rather than just being a convenient disinfectant. Emerging experimental evidence supports the role of some of these responses in the pathogenesis of the infections $A$. baumannii causes in humans and its capacity to resist antibiotics and host response effectors.

\section{Keywords}

antibiotics; ethanol; indole-3-acetic acid; iron; motility; proteomics; reactive species; transcriptomics

\begin{abstract}
The Gram-negative coccobacillus Acinetobacter baumannii is an emerging bacterial pathogen typically affecting critically ill patients with breaches in the skin or the respiratory tract [1]. A. baumannii has been shown to cause nosocomial burn infections, secondary meningitis, systemic infections, urinary tract infections, pneumonia and necrotizing fasciitis [1-3]. Severe wound infections in military personnel deployed in Iraq or Afghanistan have also been linked to $A$. baumannii [4]. Reports of $A$. baumannii infections are continually increasing [5], with Acinetobacter species being the only Gram-negative bacteria to significantly increase in prevalence as the causative agent of pneumonia in US intensive care units [6]. Treatment of $A$. baumannii has proven difficult due to its ability to upregulate or acquire genes conferring antibiotic resistance and the paucity of literature describing the virulence factors and mechanisms utilized by this pathogen to cause disease. Properties associated with the pathogenicity of $A$. baumannii are, therefore, of particular interest as the emergence of multiple antibiotic-resistant isolates has made it one of the most problematic nosocomial pathogens of the last 15 years [1]. As of 2008, A. baumannii was the fifth most common Gram-negative nosocomial pathogen preceded by Escherichia coli, Pseudomonas aeruginosa, Klebsiella pneumoniae and Enterobacter species, respectively [7].
\end{abstract}

\footnotetext{
(C) 2013 Future Medicine Ltd

*Author for correspondence: Tel.: +1 5135295424 • Fax: +1 5135292431 • actisla@ miamioh.edu.

Financial \& competing interests disclosure

The authors have no other relevant affiliations or financial involvement with any organization or entity with a financial interest in or financial conflict with the subject matter or materials discussed in the manuscript apart from those disclosed.

No writing assistance was utilized in the production of this manuscript.
} 
In addition to its widely described antibiotic-resistance properties, $A$. baumannii has the ability to persist in the human host and medical environment, where it encounters a plethora of stressors. These stressors include iron, an essential micronutrient for almost all living cells and organisms that causes severe cell damage when not properly controlled, and chemical antiseptics normally used in human medicine, such as ethanol, which cause cell damage and induce a global protective response. When compounded with its multidrugresistant phenotype, the unique properties of $A$. baumannii that allow it to persist in varying harsh conditions, including conditions of iron depletion and oxidative stress, exemplify the impact of this bacterium as a serious threat to human health worldwide. A relatively small number of reports have described the responses of $A$. baumannii to iron starvation and oxidative stress, as well as to other stressors associated with its virulence. This review presents an account of the most recent findings related to the responses of $A$. baumannii to these stressful conditions, which are most likely relevant to the virulence of this pathogen and its interaction with the medical environment and human host.

\section{Iron stress-related responses}

Active iron acquisition allows $A$. baumannii to grow under iron-limiting conditions, such as those found in the human host and in aqueous solutions used in medicine. In the human host, iron is sequestered by ferritin, transferrin, lactoferrin and heme, lowering the availability of free iron to concentrations as low as $10^{-18} \mathrm{M}$, a concentration that is below the levels needed for bacteria to persist in the environment and cause infection in humans [8]. Sequestration of iron by high-affinity iron chelators serves as an innate host defense mechanism against invading pathogens, as well as a mechanism that decreases the risk of oxidative damage that can result from the presence of excess free iron $[9,10]$. A successful bacterial pathogen must, therefore, survive by competing with the host for iron through the expression of iron-acquisition systems [8]. These systems include the production of highaffinity iron chelators known as siderophores, which are secreted into the extracellular milieu. After binding iron, the ferric siderophore complexes are internalized via specific membrane receptors and membrane permease systems that deliver this metal into the cytosol, where it is used for essential cellular functions. Bacteria also acquire iron by directly binding host iron-chelating products, such as transferrin, lactoferrin, hemoglobin and heme. Furthermore, some bacteria acquire $\mathrm{Fe}^{3+}$ by mechanisms in which iron is not complexed to a chelator, as well as by transport systems that are specific for $\mathrm{Fe}^{2+}$, a species that is more abundant under anaerobic conditions $[8,11]$.

In the case of the A. baumannii ATCC $19606^{\mathrm{T}}$ strain, iron requirements under limiting conditions are met by the production and utilization of acinetobactin [12-14]. Analyses of acinetobactin biosynthesis and transport isogenic mutants demonstrated that this highaffinity catechol-hydroxamate siderophore (Figure 1) is critical for the ability of this strain to grow in different bacteriological media supplemented with synthetic iron chelators, such as 2,2'-dipyridyl, as well as to persist in human alveolar epithelial cells, and infecting and killing Galleria mellonella larvae and mice, respectively [15]. These responses were mainly due to the acinetobactin-mediated acquisition of iron from the culture media, as well as the host cells and tissues, although a possible acinetobactin-mediated cytotoxic effect should not be discounted.

Iron is also an important environmental signal that controls the expression of a wide range of cellular functions, a role that has been reflected not only by a large number of publications related to this metal, but also by recent genome-wide studies. In the particular case of $A$. baumannii, transcriptomic analysis of the ATCC 17978 strain showed that 1207 genes are differentially expressed in response to changes in extracellular free-iron concentrations [16], with 463 and 202 genes upregulated and downregulated more than twofold, respectively, 
when bacteria were cultured in the presence of the synthetic iron chelator 2,2'-dipyridyl. A relatively large proportion of upregulated genes include those coding for proteins associated with the production of low-molecular-weight high-affinity ferric-iron chelators, called siderophores, and potential cognate outer membrane receptors, including ferric uptake regulator (Fur)-regulated genes that code for the synthesis, secretion and utilization of acinetobactin (which was originally described in the A. baumannii ATCC $19606^{\mathrm{T}}$ type strain) $[12,13]$. The acinetobactin gene cluster, which plays a key role in the virulence of this strain [15], contains 18 genes divided among seven operons and includes ten bas ( $A$. baumannii acinetobactin biosynthesis) genes, six bau ( $A$. baumannii acinetobactin utilization) genes and two $\operatorname{bar}(A$. baumannii acinetobactin release) genes (Figure 2), the functions of which have been predicted based on sequence homologies or experimentally tested using appropriate isogenic insertion derivatives [12,13]. Most notably, under conditions of iron-limitation, $A$. baumannii upregulates bas $D$, a gene involved in the biosynthesis of the siderophore acinetobactin, 165-fold [16]. Experimental infections using ex vivo (A549 human alveolar epithelial cell monolayers) and in vivo (G. mellonella larvae and mice) models show that the virulence of $A$. baumannii ATCC $19606^{\mathrm{T}}$ isogenic derivatives affected in the production or utilization of acinetobactin, is significantly reduced $[15,17]$. However, the virulence-deficient phenotype of EntA, BasD and BauA mutants is reversed when animals are infected with an inoculum that contains free inorganic iron.

Taken together, these results indicate that the acinetobactin-mediated iron acquisition system plays a key role in the ability of $A$. baumannii to cause infections in a host that imposes an iron-limiting stress condition.

The ATCC 17978 iron-dependent differential gene expression also includes traits located in siderophore gene cluster 1 and 2 [16], which could code for additional siderophore-mediated iron uptake functions that remain to be characterized. This observation indicates that $A$. baumannii could respond to iron-limiting stress signals by expressing several ironacquisition systems, a hypothesis supported by the results of the genomic analysis of seven different clinical isolates that identified six gene clusters, including the acinetobactin cluster, potentially involved in the transport of ferric and ferrous iron [18]. The expression of different siderophore-mediated iron-acquisition systems by $A$. baumannii clinical isolates is further supported by the recent finding that the AYE clinical isolate produces a siderophore different from acineto-bactin, owing to a point mutation in the ent $A$ homolog, which codes for the biosynthesis of the acinetobactin precursor dihydroxybenzoic acid [17]. This phenotype indicates that $A$. baumannii AYE most likely produces a noncatechol iron chelator that remains to be characterized structurally and functionally. The transcriptomic analysis of $A$. baumannii ATCC 17978 also showed enhanced transcription of genes coding for potential siderophore efflux functions, such as $\operatorname{bar} A$ and $\operatorname{bar} B$ (Figure 2), the products of which could be essential for intracellular iron homeostasis. These siderophore secretion functions could play a key role in iron homeostasis since intracellular accumulation of highaffinity siderophores, such as acinetobactin, could result in chelation of intracellular iron. This could affect the function of iron-containing proteins involved in critical cell functions, such as electron transport and central metabolism, as well as in the reduction of the iron labile pool needed for proper cell functioning.

The transcriptomic analysis of ATCC 17978 also suggests that iron levels influence the expression of genes coding for proteins that use iron as a cofactor, such as cytochromes involved in electron transport and enzymes involved in central metabolism [16]. Similar observations were made when the $A$. baumannii ATCC $19606^{\mathrm{T}}$ iron response was analyzed using a proteomics approach [19]. This global study showed that the presence of excess free iron affects the production of proteins associated with iron transport and storage, the tricarboxylic acid cycle and lipid metabolism, as well as proteins involved in adaptation/ stress tolerance and virulence. Some of these observations were further complemented by a 
study conducted with an iron-uptake deficient insertion derivative of $A$. baumannii ATCC $19606^{\mathrm{T}}$ affected in the production of an Fe-S scaffold protein [20]. This study demonstrated that $A$. baumannii ATCC $19606^{\mathrm{T}}$ expresses an iron-regulated gene coding for an NfuA-like $\mathrm{Fe}-\mathrm{S}$ scaffold protein that plays multiple functional roles related to iron-mediated stress. This gene is also found in the genome of all $A$. baumannii sequenced strains, including the ATCC 17978 strain, in which this ortholog was annotated as A1S_0979. The A. baumannii ATCC $19606^{\mathrm{T}} \mathrm{NfuA}$-like protein is involved in intracellular iron metabolism, perhaps serving as an internal iron source and a protector of bacterial cells from oxidative stress caused by organic and inorganic peroxides when grown in vitro under iron limitation. The A. baumannii ATCC $19606^{\mathrm{T}} \mathrm{NfuA}$ ortholog also plays a role in the virulence of this pathogen when tested using the ex vivo and in vivo experimental infection models mentioned above, by protecting infecting bacteria from oxidative responses rather than providing iron to bacteria growing under iron-limited conditions imposed by cultured human alveolar epithelial cells and $G$. mellonella larvae [20]. A. baumannii also reacts to potential stress conditions created by iron overloading, which could cause cell damage owing to the particular chemistry of iron and its participation in the generation of reactive oxygen species (ROS). Free-iron-rich conditions induce gene expression and production of proteins related to intracellular iron storage, such as bacterioferritin and hemerythrin, as well as adaptation/ stress response proteins, such as GroEL and SodB, and proteins that could control the influx/ efflux of iron or participate in the metabolism of this metal, such as OmpA and OmpW $[16,19]$. Taken together, these studies provide an important overview of the role that iron plays as a key micronutrient as well as a global regulator, which promotes the expression of genes coding for iron acquisition functions only when needed, to avoid irreparable cellular damages.

While the iron-scavenging and -sequestering mechanisms utilized by $A$. baumannii have been somewhat characterized, the complete response of $A$. baumannii to conditions of iron limitation are just beginning to be elucidated. For example, although the name of the genus Acinetobacter implies lack of movement, there have been reports describing the ability of Acinetobacter strains to move on agar or agarose surfaces, with the report by Mussi et al. being one the first to describe the motility of $A$. baumannii clinical isolates on semisolid surfaces [21]. Although the cell mechanisms associated with the motility of $A$. baumannii have not been definitively established, the studies conducted by Eijkelkamp et al. and Clemmer et al. have shed some light on the genes and gene products that could be involved in bacterial motility on semisolid agarose plates [16,22]. These two studies demonstrated that iron chelation affects the motility of $A$. baumannii ATCC 17978; motility is impaired; genes encoding components involved in type IV-mediated motility of ATCC 17978 cells, such as pilB-D, pilT, pilU, com $M-O$, comL and comQ, are downregulated under iron limitation; and inactivation of pilT, which is involved in pilus retraction, impairs the motility of the A. baumannii M2 clinical strain. Interestingly, the inactivation of pilT does not result in complete abolishment of M2 motility and it is, therefore, conceivable that cell functions other than type IV pili production could contribute to A. baumannii motility. Notably, quorum sensing has been implicated in having an effect on M2 motility, as a null mutant of abal, an autoinducer synthase coding for the production of $N$-(3-hydroxydodecanoyl)-Lhomoserine lactone, is decreased in motility, an effect that is reversed upon exogenous supplementation with the purified autoinducer [22]. However, nothing is known about the mechanisms by which iron and quorum sensing control the expression of $A$. baumannii genes coding for motility functions.

Analysis of strains obtained from wounded soldiers showed that motility on semisolid surfaces is widespread, but not universal among $A$. baumannii clinical isolates [23]. Furthermore, this study demonstrated that $A$. baumannii motility is not only controlled by the free-iron status of the environment, but also other extracellular signals such as the 
presence of $\mathrm{NaCl}$, ethanol, Congo red, $\mathrm{H}_{2} \mathrm{O}_{2}$, triclosan and subinhibitory concentrations of antibiotics. All these observations indicate that $A$. baumannii motility on semisolid surfaces is indeed a highly regulated complex process that responds to a wide range of environmental and perhaps intracellular signals by mechanisms that remain to be identified and characterized. Such a regulated response could be due to pili production and assembly being a multistep process that involves several components requiring a great deal of energy, which cells will not expend unless it is needed for persistence in the human host and/or the medical environment. Thus, it is possible to speculate that skin wounds could provide an iron-rich, oxidatively stressful environment because of cell/tissue damage and host responses that may favor the migration of $A$. baumannii towards these sites. Such a response could provide essential nutrients needed for the development of severe skin infections, such as those recently described in cases of necrotizing fasciitis in which $A$. baumannii was shown to be the sole etiological agent [3]. This hypothesis is supported by the observation that the dermis of healing and nonhealing skin ulcers contains significantly higher levels of ferric ions and host iron-binding proteins than normal skin tissue [24]. Furthermore, skin wounds, particularly chronic wounds, seem to present an oxidative-stress environment to which bacteria must respond by mounting appropriate protective responses. Although these are attractive hypotheses, the role of $A$. baumannii iron-acquisition and motility functions, as well as the participation of protective oxidative stress responses in the pathogenesis of severe skin infection, remain to be elucidated using appropriate isogenic derivatives and experimental models.

In contrast to the observations related to the differential expression of genes potentially coding for type IV pili, the effect of iron limitation on the expression of the type I pili assembly system is only limited to the ATCC 17978 gene coding for the CsuC protein [16], the chaperone component of the CsuA/BABCDE pilus assembly system. This chaperoneusher pili assembly system proved to be essential for $A$. baumannii ATCC $19606^{\mathrm{T}}$ cells to attach to and form biofilms on abiotic surfaces [25]. Although the transcriptomic results are in accordance with the observation that iron does not alter the formation of biofilms on abiotic surfaces by ATCC 17978, these data are not in agreement with the observations previously reported showing that the addition of synthetic iron chelators, such as 2,2'dipyridyl or ethylenediamine-di-( $O$-hydroxyphenyl) acetic acid, enhance the formation of ATCC $19606^{\mathrm{T}}$ biofilms on plastic [25]. This disparity could be due to different strains being used in these two studies (ATCC $19606^{\mathrm{T}}$ vs ATCC 17978), differences in culturing conditions or differences in the expression of the csu operon between these two $A$.

baumannii strains. To further explain these differences, the csu operon has been suggested to be inactive in ATCC 17978 and, in addition to pili, other iron-regulated products have been implicated in adherence and biofilm formation [26].

\section{Growth-phase stress-related responses}

Bacteria respond not only to stress signals caused by the addition/presence of particular extracellular stressors, such as free iron, but also to changes in intracellular stressors that occur as a consequence of nutrient consumption and waste generation as they grow in closed culture systems normally used in laboratory studies. A recent report in which the investigators combined 2D gel electrophoresis-MS with isobaric tags for relative and absolute quantitation (iTRAQ) liquid chromatography-MS/MS, showed that there are significant changes in the proteome of $A$. baumannii ATCC 17978 cells as they progress through the different growth stages when cultured in rich medium such as Mueller-Hinton [27]. This global study showed that there are not only significant changes in the production of 107 proteins as ATCC 17978 cells transition from the exponential into the late-stationary growth phase, but there are also relevant protein changes due to oxidative-dependent protein modification processes that occur after proteins are produced. Although growth into the 
stationary phase results in an overall downproduction of proteins involved in gene transcription and translation (with the exception of elongation factors), ribosome biogenesis, cell-division processes, and amino acid and lipid transport and metabolism, there is a clear induction in the production of proteins involved in putative signaling functions, the biogenesis of the bacterial cell wall and in redox reactions that are related to oxidative stressors.

A. baumannii 17978 also responds to potential cell wall damage caused by growth into the stationary phase by increasing the production of NlpE, an outer membrane lipoprotein that activates the Cpx signaling pathway. This pathway relates to stress caused by damage to the cell envelope due to a wide range of extracellular conditions and interactions with surfaces and other cells during biofilm formation [28]. Interestingly, a comparative proteomic analysis of the ATCC $19606^{\mathrm{T}}$ strain with the multidrug-resistant strain A6, isolated from hospitalized French patients, showed an association between the higher ability of A6 to form biofilms on abiotic surfaces and an increase in the production of NlpE when compared with the response observed in the relatively sensitive ATCC $19606^{\mathrm{T}}$ type strain [29]. Taken together, these observations suggest a role for NlpE in sensing and inducing responses to different stressors, including the interaction with and the formation of biofilm on abiotic surfaces, which may result in damaging alterations to the $A$. baumannii cell wall. However, these possibilities remain to be confirmed by examining the responses of proper isogenic strains affected in the production of NlpE.

Envelope biogenesis and production of outer membrane proteins are also significantly affected as cells grow into the stationary phase [27]. There is a significant increase in the production of A1S_0820, a protein containing the LysM domain that could participate in the remodeling of the cell wall as cells grow into the stationary phase. The LysM domain has been associated with the retention of proteins in the bacterial cell wall by their noncovalent attachment to the peptidoglycan layer. Most of the LysM-containing proteins function as peptidoglycan hydrolases with various substrate specificities [30]. There is also an increased production of Omp38 (also called OmpA) and a precursor of CarO in late-stationary phase cells. OmpA plays a key role in the ability of $A$. baumannii to interact with solid surfaces, particularly biotic surfaces and host products such as fibronectin, and causes apoptosis of eukaryotic cells [31-33]. CarO is an outer membrane protein implicated in antibiotic resistance and ornithine transport [34]. These results indicate that cell growth and cellsurface interactions create cell stresses that need proper control for $A$. baumannii to persist under different environmental conditions.

The two methods the investigators used to determine the global $A$. baumannii protein responses at different growth phases [27] also showed that there is a significant increase in the production of proteins, such as bacterioferritin and heme-containing flavoproteins, which protect cells from damage resulting from an intracellular accumulation of free-iron in older bacteria. There is also a relevant overproduction of proteins related to oxidative stress caused by ROS, mainly $\mathrm{O}_{2}{ }^{-}$and $\mathrm{OH}^{-}$, as well as reactive nitrogen intermediates (RNI), mainly $\mathrm{ONOO}^{-}$. These overproduced proteins include detoxifying enzymes such as putative oxidoreductase, alkyl hydroperoxide reductase (an antioxidant protein belonging to the glyoxalase I superfamily), glutathione peroxidase, catalase-peroxidase, catalase HPII and two universal stress proteins. The latter proteins play a role in the ability of bacteria, such as E. coli, to resist $\mathrm{O}_{2}^{-}$-generating agents and express motility and adherence functions [35]. Interestingly, the overproduction of these oxidative-stress response proteins correlates with a concomitant increase in the production of ROS and RNI compounds in A. baumannii ATCC 17978 stationary phase cells. This oxidative stress-induced response also provides stationary-phase cells with protection from $\mathrm{H}_{2} \mathrm{O}_{2}$, menadione (an $\mathrm{O}_{2}{ }^{-}$donor) and sodium nitroprusside (a NO donor) when added to the culture medium used to grow ATCC 17978 
cells. The enhanced $A$. baumannii oxidative-stress response detected in stationary-phase cells also includes the overproduction of macromolecule repair proteins, such as the chaperone DnaK that repairs protein oxidative damage, as well as the overproduction of proteins that protect and repair DNA damage caused by ROS and RNI compounds, such as IHF and RecA. Interestingly, RecA proved to be critical for the ability of $A$. baumannii to resist DNA damage from heat shock and desiccation, conditions that may induce the production of the cell damaging compounds listed above [36]. The response of $A$. baumannii to desiccation is of particular concern considering the distinct ability of this pathogen to survive for more than 30 days on dry abiotic surfaces, a response that is more common among Gram-positive than Gram-negative bacteria [37,38]. Interestingly, desiccation also results in significant changes in cell shape and structure. A. baumannii-desiccated cells are more rounded and have thicker cell walls than control cells [39]. Furthermore, the ability of clinical isolates to survive on dry surfaces seems to relate to their capacity to form biofilms on abiotic surfaces [40], a conclusion that remains to be confirmed by testing the responses of isogenic clinical strains affected in adherence and/or biofilm formation. Such a cell response to a stressor, which may induce a protective response against oxidative damage owing to the described RecA role, could explain the persistence of this pathogen in medical settings under unfavorable conditions on abiotic surfaces serving as potential nosocomial reservoirs [41].

Although the proteins associated with oxidative-stress responses are believed to be confined to the bacterial cytoplasm, analysis of the extracellular proteome of $A$. baumannii AbH120A2, a highly invasive multidrug-resistant isolate that infected more than 300 patients in Madrid, Spain [42], indicates that several of these proteins are secreted during early stationary phase. This study revealed the presence of oxidoreductase, catalase, thioredoxin, flavohemoprotein and superoxide dismutase in the free soluble extracellular protein fraction, while alkyl hydroperoxide reductase and $\mathrm{Cu} / \mathrm{Zn}$ superoxide dismutase are present in both the free soluble extracellular protein and the outer membrane vesicle fractions. Furthermore, incubation of A. baumannii AbH120-A2 at the exponential growth phase with concentrated free soluble extracellular proteins obtained from this strain during early stationary-phase growth provided significant protection from oxidative damage caused by $\mathrm{H}_{2} \mathrm{O}_{2}$ and allowed bacteria to better survive macrophage attack. All these observations indicate that both intracellular and extracellular detoxification processes are not only induced during growth, but are also important in the ability of $A$. baumannii to resist oxidative responses mounted by the host during infection.

Proteins such as aconitate hydratase-1, superoxide dismutase, OmpA, OmpW, bacterioferritin, $\mathrm{CarO}$, the ribosomal protein $\mathrm{L} 3$ and putative flavohemoproteins are overproduced by cells in stationary phase or by those cultured under iron-rich conditions. These observations indicate that $A$. baumannii cells react to two stressors with common or overlapping protective responses. It is also possible to speculate that although the responses reported by Soares et al. may not be mounted during infection, as bacteria may not reach the stationary-phase conditions achieved under laboratory conditions [27], the observations described in that report could be biologically relevant. These responses may indeed reflect the ability of $A$. baumannii to withstand host defense responses, including the oxidative damage caused by macrophages during the pathogenesis of the infections it causes in the human host. Such a possibility is supported by the observation that inactivation of RecA, a key protein that protects DNA from damage caused by oxidative stress, significantly reduces the virulence of $A$. baumannii when tested using human macrophages and an experimental mouse infection model [36]. 


\section{Effects of stress responses on antibiotic resistance}

The response of $A$. baumannii to the presence of antibiotics under iron limitation and oxidative stress conditions is of particular interest considering the ability of this pathogen to express resistance to a wide range of antibiotics used in human medicine [43]. In general, the multidrug-resistance phenotype of $A$. baumannii is attributed to its capacity to acquire and overexpress genes coding for a wide range of antibiotic resistance functions, as well as its natural resistance owing to particular membrane permeability properties [44]. However, it is possible that the antibiotic resistance of this pathogen is not only due to the presence or gain of genes coding for resistance, but also their enhanced expression under particular stress conditions that $A$. baumannii encounters in the human host, such as iron restriction. This possibility is supported by transcriptome [16] and proteome [19] global analyses showing the upregulation and consequent overproduction of $c a r O$ and its translation product in A. baumannii cells cultured under iron limitation. This is an interesting protein that could play dual roles in the pathobiology of $A$. baumannii. CarO is associated with carbapenem resistance, probably owing to its substrate-independent pore-forming properties, as well as its role in the transport of amino acids such as 1-ornithine [34,45]. This amino acid is typically found in low levels in blood plasma; however, following severe skin trauma, the amount of orinithine increases in the plasma [46]. Considering this observation, Mussi et al. have suggested that $A$. baumannii may use increases in ornithine to find a portal of entry into a compromised host [34]. These results indicate that iron limitation, a condition experienced by most pathogens in the human host, is an important stressor that not only affects resistance to carbapenem, which is considered a resistance sentinel event [47], but also causes the overproduction of $\mathrm{CarO}$, allowing $A$. baumannii to sense portals of entry into the host and establish an active infection.

Iron limitation also results in the upregulation of genes encoding multidrug efflux proteins that belong to the resistance-nodulation-division (RND) family, such as AdeA, AdeB, AdeK and AdeI [16]. RND efflux pumps utilize the proton motive force to drive the expulsion of antimicrobials [48] leading to resistance against aminoglycosides, $\beta$-lactams, chloramphenicol, erythromycin and tetracycline [49]. There are three RND efflux pumps currently known to be utilized by A. baumannir: AdeABC [49], AdeFGH [50] and AdeIJK [51]. These pumps include three components: a membrane fusion protein (AdeA, AdeF or AdeI), a multidrug transporter (AdeB, AdeG or AdeJ) and an outer membrane protein (AdeC, AdeH or AdeK), all of which function in a coordinated fashion to allow antibiotics to be removed from the cytoplasm without accumulating in the periplasm [49,52]. The AdeABC efflux pump is regulated by AdeRS, a two-component regulatory system composed of a sensor kinase (AdeS) and a response regulator (AdeR) [53]. Interestingly, AdeS activates or inactivates AdeR, which controls expression of the AdeABC efflux pump in response to environmental conditions [53]. In the case of the AdeFGH efflux system, AdeL, a LysR-type regulator, controls the expression of this system, while the potential regulator controlling the expression of the AdeIJK system has not been clearly defined. Although the transcriptomic data reported by Eijkelkamp et al. indicate a potential regulatory role of iron in the expression of RND-mediated resistance functions by mechanisms that remain to be characterized [16], an effect of this metal on antibiotic minimal inhibitory concentrations and bacterial susceptibility under iron-limiting conditions, which would mimic those found in the human host, remains an interesting possibility that warrants further study.

Oxidative stress responses also relate to the ability of $A$. baumannii to resist antibiotics. On one hand, induction of oxidative responses, such as those observed in late ATCC 17978 stationary-phase cells, results in an increase in resistance to $\beta$-lactams, colistin and quinolones [27], which represent the three major classes of antibiotics used to treat $A$. 
baumannii human infections. On the other hand, the presence of different antibiotics at subinhibitory concentrations induces $A$. baumannii protective cell responses, which include the overproduction of the major molecular chaperones DnaK and GroEL. These two proteins, which protect proteins from misfolding, aggregation or degradation due to damage caused by oxidative and heat shock stresses, were overproduced when $A$. baumannii cells were cultured at $50^{\circ} \mathrm{C}$ or exposed to subinhibitory concentrations of streptomycin [54]. Furthermore, bacteria pretreated at $45^{\circ} \mathrm{C}$ for $30 \mathrm{~min}$ proved to be more thermotolerant and resistant to antibiotics than bacteria cultured under standard conditions at $37^{\circ} \mathrm{C}$. In addition, incubation of the $A$. baumannii multidrug-resistant strain RS4 in the presence of different antibiotics resulted in a significant increase in the production of DnaK. These results indicate that $A$. baumannii uses common responses to protect cells from damage caused by either stressor, temperature or antibiotics, or antibiotics with different primary targets. This response is congruent with the interesting observation that bactericidal antibiotics work not only by interacting with specific primary targets, such as ribosomes, DNA gyrase and penicillin-binding proteins, but also by generating $\mathrm{OH}^{-}$radicals via Fenton chemistry, dependent on the tricarboxylic acid cycle and the damage of Fe-S cluster proteins, which cause generalized macromolecular damage that results in bacterial death [55]. In the particular case of $A$. baumannii, it was recently shown that the bactericidal activity of polymyxins, such as polymixin $\mathrm{B}$ and colistin, depends on the production of $\mathrm{OH}^{-}$radicals [56]. Bacteria respond to this oxidative stress by inducing a SOS response in which RecA and chaperones are responsible for repairing DNA damage and correcting protein misfolding, respectively. Interestingly, analysis of $A$. baumannii European clone II isolates harboring variants of the AbaR-resistant island revealed the presence of an intact uspA gene, which codes for a universal stress protein homolog. As aforementioned, Usp proteins were shown to play a role in bacterial responses to oxidative stress [35]. Whether the presence of a gene coding for such a function within resistant islands provides a selective advantage to this pathogen to persist better in the presence of ROS-generating antibiotics is an interesting possibility that remains to be determined.

\section{Ethanol stress-related responses}

The presence and persistence on hospital fomites as well as on human sites, such as the skin and throat, facilitates the transmission of $A$. baumannii within nosocomial settings and the pathogenesis of infections mostly among compromised patients. Owing to this and the severity of $A$. baumannii infections, strict hygiene and disinfection procedures are normally implemented in hospitals and medical offices, including the use of ethanol-based hand rub, gel and foam disinfectants. However, ethanol has an impact in the development of $A$. baumannii human infections, as their frequency and severity increases significantly among alcoholic patients $[57,58]$. Furthermore, ethanol misuse and its volatile properties may hinder the antiseptic properties of this widely used disinfectant. Thus, owing to its effects on the human host or the misuse of ethanol-based disinfectants [59], the continuous presence of ethanol could represent an important environmental factor that affects the epidemiology of the diseases $A$. baumannii causes in hospitalized patients as well as its physiology and virulence properties [60]. This hypothesis is supported by the observation that the presence of low concentrations of ethanol, particularly in nutrient-restricted media, favors the growth of this pathogen and induces the production of OmpA [61]. This protein, which plays a role in the virulence of this pathogen and its interaction with human cells, has emulsifying activities that may help this bacterium to use hydrocarbons, including ethanol itself, as a nutrient source [31,32]. The presence of low amounts of ethanol in the culture medium also increases the salt tolerance and the virulence of $A$. baumannii when tested using Caenorhabditis elegans in experimental infections [62]. Taken together, these observations indicate that ethanol is a stressor that could have a global effect on the physiology and pathobiology of $A$. baumannii. Accordingly, a recent global transcriptomic analysis of $A$. 
baumannii ATCC 17978 revealed the differential transcription of a large number of genes in cells cultured in the presence of $1 \%$ ethanol [63]. A similar observation was made when the A. baumannii ATCC 17978 response to the presence of ethanol was examined using a proteomics approach that showed the differential production of 34 proteins when cells were cultured in the presence or absence of ethanol [64]. Although the increased transcription of some genes correlates with an increased production of cognate proteins, the differential production of some ethanol-regulated proteins seems to be controlled by post-transcriptional mechanisms that remain to be characterized. Overall, these two global approaches showed that the presence of ethanol induces a differential response in gene expression and protein production related to central metabolic functions associated with ethanol/acetate assimilation, as well as responses related to general metabolism, energy production and nucleotide and protein biosynthesis. Ethanol also induces a strong adaptation/stress response that includes the differential expression of genes and the production of proteins related to oxidative stress, such as superoxide dismutase, glutathione peroxidase and alkyl hydroperoxide reductase, in addition to proteases and chaperones, such as GroEL, which protect cells from the perturbations caused by protein misfolding. These responses resemble those induced by the overproduction of ROS and RNS as cells age as well as the presence of antibiotics previously described; an observation that indicates, at least in part, different stressors induce common $A$. baumannii protective responses.

Ethanol also induces an overall lipid biosynthesis response that may reflect a cell membrane adaptation to the presence of this stressor or a response that could induce the formation of multicellular structures, such as biofilms, which protect bacteria from environmental injuries and host-mediated damages. The latter possibility is supported by the ethanol-mediated enhanced expression of genes and the production of proteins associated with carbohydrate biosynthesis detected by transcriptomic [63] and proteomic analyses [64]. Lipids and carbohydrates play a critical role in bacterial biofilm formation and development [65]. Interestingly, our study also showed that the presence of ethanol induces a significant increase in the formation of biofilms on plastic under static culture conditions. Figure 3A demonstrates that the presence of ethanol induces the formation of more dense and complex biofilms on plastic than those formed in the absence of ethanol at the liquid-air interface. Furthermore, the carbohydrate content of biofilms formed in the presence of ethanol was higher when compared with that of biofilms formed in the absence of ethanol.

The increased attachment to and formation of biofilm on abiotic surfaces, induced by ethanol, contrasts with the drastic reduction in motility of A. baumannii ATCC 17978 on agarose swimming plates containing ethanol (Figure 3B). Although the association between reduced motility and increased biofilm formation is not unexpected, considering that nonmotile cells have a higher chance of adhering to and forming biofilms on solid substrates [66], the effect of ethanol on the expression of these two phenotypes seems novel since such responses have not been previously associated with the cell toxicity of ethanol to $A$.

baumannii or other major bacterial pathogens. Such a response seems to be logical if one considers that planktonic motile bacteria would be more exposed and, therefore, more susceptible to ethanol toxicity and stress than sessile bacteria. The latter type of cells would be more protected from ethanol, not only because of their encasement within the biofilm matrix and multicellular structures, but also because the particular physiology of sessile cells could render them more resistant to ethanol-induced cell damages.

The acetogenic response induced by the presence of ethanol also results in a significant $\mathrm{pH}$ reduction of culture supernatants [64]. We attributed such a pH change to the accumulation of acetate that could result in the consequent production of acid derivatives such as indole-3acetic acid (IAA), which has a profound effect on bacterial gene expression and physiology. IAA increases production of lipopolysaccharides and exopolymeric substances, enhances 
biofilm formation and induces stress resistance [67-70]. These observations prompted us to examine whether the presence of ethanol in the culture medium affects the production and secretion of IAA. Our analysis showed that bacteria cultured in nutrient-rich or -limited media in the presence of ethanol produce more IAA than cells cultured in the absence of this alcohol, a response that is not limited to the ATCC 17978 strain; ten different A. baumannii clinical isolates showed a similar response. It is also important to note that our experimental infection assays, using $G$. mellonella as a host, produced results similar to those obtained with $C$. elegans and Dictyostelium discoideum [62,71]; the virulence of $A$. baumannii ATCC 17978 is significantly enhanced when grown in the presence of ethanol.

Taken together, these observations indicate that ethanol is a stressor that plays a critical role in the virulence of $A$. baumannii and the pathogenesis of human infections. It induces physiological and cellular responses that protect bacteria from cellular damage via several mechanisms and promotes the production of IAA, a compound that not only globally affects bacterial physiology, but also has the potential for affecting important host-pathogen interactions. IAA, a plant growth hormone, plays a critical role in the interaction of plants and bacteria, including processes associated with pathogenesis and phytostimulation [72], some of which may resemble those occurring during the interaction of $A$. baumannii with the human host. Interestingly, IAA is an efficient carbon source for $A$. baumannii [73] and human fluids contain IAA as well as related derivatives such as 5-hydroxyindole-3-acetic acid [74-76]. Thus, ethanol seems to influence the virulence of $A$. baumannii and the pathogenesis of the infections it causes in humans through a complex network of bacterial and host effects and responses. At the level of the human host, ethanol makes it more susceptible to infections. At the level of the pathogen, ethanol induces a complex cell protective stress response, as well as the expression of factors affecting key host-pathogen interactions, such as adherence and biofilm formation; ethanol even favors bacterial growth by providing an unpredicted, convenient carbon source that is abundant in current medical settings.

\section{Future perspective}

A. baumannii is considered an emerging multidrug-resistant Gram-negative bacterial pathogen that causes severe human infections worldwide. Most of the research conducted to date focuses upon the taxonomy and epidemiology of $A$. baumannii and the infections it causes in compromised patients, as well as the mechanisms by which this pathogen resists a wide range of antibiotics. However, less work has been conducted concerning the virulence factors and stress responses expressed by $A$. baumannii, although some progress has been achieved in recent years. This review describes recent advances in the understanding of $A$. baumannii molecular and cellular responses to a variety of stressors, including iron limitation, oxidative stress, ethanol, antibiotics, heat shock, desiccation and growth, with the underlying theme of how these responses participate in this pathogen's ability to persist in medical settings and resist a wide range of antimicrobials as well as the stressful conditions imposed by the human host. All these studies have shown that $A$. baumannii is capable of sensing and responding to different stressors by mounting complex protective responses that, in some cases, have significant overlaps. However, knowledge is still lacking when compared with better-characterized human opportunistic pathogens, such as $P$. aeruginosa. Although finding stress responses already described in other pathogens is a possibility, the particular physiology and pathobiology of $A$. baumannii could result in novel and exciting observations. These observations could be made by using interdisciplinary approaches that exploit continued advances in technology, such as global genomic and gene expression analyses combined with convenient high-throughput random mutagenesis systems. All these approaches, together with proper experimental models that mimic the different types of infections $A$. baumannii causes in humans, should provide important basic information 
related to the pathophysiology of this bacterium, including its ability to cause different types of human infections, some of which were not reported until recently. This knowledge will also facilitate the development of novel and/or alternative drugs and therapeutic methods needed to treat the recalcitrant infections caused by $A$. baumannii multidrug-resistant isolates.

\section{Acknowledgments}

The authors' work and results reported in this review were supported by funds from Public Health AI070174 and NSF 0420479, CHE0839233 grant awards and Miami University research funds.

\section{References}

Papers of special note have been highlighted as:

- of interest

- of considerable interest

1. Peleg AY, Seifert H, Paterson DL. Acinetobacter baumannii: emergence of a successful pathogen. Clin. Microbiol. Rev. 2008; 21(3):538-582. [PubMed: 18625687]

2. Bergogne-Berezin E, Towner KJ. Acinetobacter spp. as nosocomial pathogens: microbiological, clinical, and epidemiological features. Clin. Microbiol. Rev. 1996; 9(2):148-165. [PubMed: 8964033]

3. Charnot-Katsikas A, Dorafshar AH, Aycock JK, David MZ, Weber SG, Frank KM. Two cases of necrotizing fasciitis due to Acinetobacter baumannii. J. Clin. Microbiol. 2009; 47(1):258-263. [PubMed: 18923009]

4. Davis KA, Moran KA, McAllister CK, Gray PJ. Multidrug-resistant Acinetobacter extremity infections in soldiers. Emerg. Infect. Dis. 2005; 11(8):1218-1224. [PubMed: 16102310]

5. Peleg AY, Hooper DC. Hospital-acquired infections due to gram-negative bacteria. N. Engl. J. Med. 2010; 362(19):1804-1813. [PubMed: 20463340]

6. Gaynes R, Edwards JR. Overview of nosocomial infections caused by Gram-negative bacilli. Clin. Infect. Dis. 2005; 41(6):848-854. [PubMed: 16107985]

7. Hidron AI, Edwards JR, Patel J, et al. NHSN annual update. antimicrobial-resistant pathogens associated with healthcare-associated infections: annual summary of data reported to the National Healthcare Safety Network at the Centers for Disease Control and Prevention, 2006-2007. Infect. Control Hosp. Epidemiol. 2008; 29(11):996-1011. [PubMed: 18947320]

8. Crosa, JH.; Mey, AR.; Payne, SM. Iron Transport in Bacteria. Washington, DC, USA: ASM Press; 2004.

9. Weinberg ED. Iron withholding: a defense against infection and neoplasia. Physiol. Rev. 1984; 64(1):65-102. [PubMed: 6420813]

10. Weinberg ED. Iron availability and infection. Biochem. Biophys. Acta. 2009; 1790(7):600-605. [PubMed: 18675317]

11. Skaar EP. The battle for iron between bacterial pathogens and their vertebrate hosts. PLoS Pathog. 2010; 6(8):e1000949. [PubMed: 20711357]

12. Dorsey CW, Tomaras AP, Connerly PL, Tolmasky ME, Crosa JH, Actis LA. The siderophoremediated iron acquisition systems of Acinetobacter baumannii ATCC 19606 and Vibrio anguillarum 775 are structurally and functionally related. Microbiology. 2004; 150(Pt 11):36573667. [PubMed: 15528653]

13. Mihara K, Tanabe T, Yamakawa Y, et al. Identification and transcriptional organization of a gene cluster involved in biosynthesis and transport of acinetobactin, a siderophore produced by Acinetobacter baumannii ATCC 19606 ${ }^{\mathrm{T}}$. Microbiology. 2004; 150(Pt 8):2587-2597. [PubMed: 15289555] 
14. Yamamoto S, Okujo N, Sakakibara Y. Isolation and structure elucidation of acinetobactin, a novel siderophore from Acinetobacter baumannii. Arch. Microbiol. 1994; 162(4):249-252. [PubMed: 7802543]

15. Gaddy JA, Arivett BA, McConnell MJ, Lopez-Rojas R, Pachón J, Actis LA. Role of acinetobactinmediated iron acquisition functions in the interaction of Acinetobacter baumannii ATCC $19606^{\mathrm{T}}$ with human lung epithelial cells, Galleria mellonella caterpillars and mice. Infect. Immun. 2012; 80(3):1015-1024. [PubMed: 22232188]

16. Eijkelkamp BA, Hassan KA, Paulsen IT, Brown MH. Investigation of the human pathogen Acinetobacter baumannii under iron limiting conditions. BMC Genomics. 2011; 12:126. [PubMed: 21342532] . Global analysis of Acinetobacter baumannii ATCC 17978 showing differential gene expression in response to iron limitation. Microarray data show that iron-limiting conditions result in the upregulation of genes coding for siderophore production and utilization, but negatively affect cell motility on semisolid media..

17. Penwell WF, Arivett BA, Actis LA. The Acinetobacter baumannii entA gene located outside the acinetobactin cluster is critical for siderophore production, iron acquisition and virulence. PLoS One. 2012; 7(5):e36493. [PubMed: 22570720]

18. Antunes LC, Imperi F, Towner KJ, Visca P. Genome-assisted identification of putative ironutilization genes in Acinetobacter baumannii and their distribution among a genotypically diverse collection of clinical isolates. Res. Microbiol. 2011; 162(3):279-284. [PubMed: 21144895]

19. Nwugo CC, Gaddy JA, Zimbler DL, Actis LA. Deciphering the iron response in Acinetobacter baumannii : a proteomics approach. J. Proteomics. 2011; 74(1):44-58. [PubMed: 20692388] . Proteomic analysis of A. baumannii ATCC $19606^{\mathrm{T}}$ in response to iron, which promotes the differential production of proteins involved in different cellular processes including stress tolerance.

20. Zimbler DL, Park TM, Arivett BA, et al. Stress response and virulence functions of the Acinetobacter baumannii NfuA Fe-S scaffold protein. J. Bacteriol. 2012; 194(11):2884-2893. [PubMed: 22467784] . memonstrates that an A. baumannii ATCC $19606^{\mathrm{T}}$ NfuA-like Fe-S scaffold protein protects bacterial cells from oxidative damage, but does not function as an iron source under iron-limiting conditions imposed by human alveolar epithelial cells and Galleria mellonella larvae.

21. Mussi MA, Gaddy JA, Cabruja M, Viale AM, Rasia R, Actis LA. Motility, virulence and biofilm formation by the human pathogen Acinetobacter baumannii are affected by blue light. J. Bacteriol. 2010; 192(24):6336-6345. [PubMed: 20889755] . - One of the first reports to describe the motile phenotype of $A$. baumannii, which was believed to be nonmotile, on semisolid surfaces. Light affects the expression of this phenotype as well as biofilm formation and virulence.

22. Clemmer KM, Bonomo RA, Rather PN. Genetic analysis of surface motility in Acinetobacter baumannii. Microbiology. 2011; 157(Pt 9):2534-2544. [PubMed: 21700662]

23. McQueary CN, Kirkup BC, Si Y, et al. Extracellular stress and lipopolysaccharide modulate Acinetobacter baumannii surface-associated motility. J. Microbiol. 2012; 50(3):434-443. [PubMed: 22752907]

24. Yeoh-Ellerton S, Stacey MC. Iron and 8-isoprostane levels in acute and chronic wounds. J. Invest. Dermatol. 2003; 121(4):918-925. [PubMed: 14632213]

25. Tomaras AP, Dorsey CW, Edelmann RE, Actis LA. Attachment to and biofilm formation on abiotic surfaces by Acinetobacter baumannii: involvement of a novel chaperone-usher pili assembly system. Microbiology. 2003; 149(Pt 12):3473-3484. [PubMed: 14663080]

26. McConnell MJ, Actis L, Pachón J. Acinetobacter baumannir: human infections, factors contributing to pathogenesis and animal models. FEMS Microbiol. Rev. 2013; 37(2):130-155. [PubMed: 22568581]

27. Soares NC, Cabral MP, Gayoso C, et al. Associating growth-phase-related changes in the proteome of Acinetobacter baumannii with increased resistance to oxidative stress. J. Proteome Res. 2010; 9(4):1951-1964. [PubMed: 20108952] . Demonstrates significant changes in the proteome of $A$. baumannii ATCC 17978 as it transitions from exponential-phase into late-stationary-phase growth, including the induction of putative virulence factors, signaling proteins and proteins associated with protection from oxidative stress. 
28. Dorel C, Lejeune P, Rodrigue A. The Cpx system of Escherichia coli, a strategic signaling pathway for confronting adverse conditions and for settling biofilm communities? Res. Microbiol. 2006; 157(4):306-314. [PubMed: 16487683]

29. Siroy A, Cosette P, Seyer D, et al. Global comparison of the membrane subproteomes between a multidrug-resistant Acinetobacter baumannii strain and a reference strain. J. Proteome Res. 2006; 5(12):3385-3398. [PubMed: 17137340]

30. Buist G, Steen A, Kok J, Kuipers OP. LysM, a widely distributed protein motif for binding to (peptido)glycans. Mol. Microbiol. 2008; 68(4):838-847. [PubMed: 18430080]

31. Choi CH, Lee EY, Lee YC, et al. Outer membrane protein 38 of Acinetobacter baumannii localizes to the mitochondria and induces apoptosis of epithelial cells. Cell Microbiol. 2005; 7(8):11271138. [PubMed: 16008580]

32. Gaddy JA, Tomaras AP, Actis LA. The Acinetobacter baumannii 19606 OmpA protein plays a role in biofilm formation on abiotic surfaces and the interaction of this pathogen with eukaryotic cells. Infect. Immun. 2009; 77(8):3150-3160. [PubMed: 19470746]

33. Smani Y, Mcconnell MJ, Pachón J. Role of fibronectin in the adhesion of Acinetobacter baumannii to host cells. PLoS One. 2012; 7(4):e33073. [PubMed: 22514602]

34. Mussi MA, Relling VM, Limansky AS, Viale AM. CarO, an Acinetobacter baumannii outer membrane protein involved in carbapenem resistance, is essential for l-ornithine uptake. FEBS Lett. 2007; 581(29):5573-5578. [PubMed: 17997983]

35. Nachin L, Nannmark U, Nystrom T. Differential roles of the universal stress proteins of Escherichia coli in oxidative stress resistance, adhesion, and motility. J. Bacteriol. 2005; 187(18): 6265-6272. [PubMed: 16159758]

36. Aranda J, Bardina C, Beceiro A, et al. Acinetobacter baumannii RecA protein in repair of DNA damage, antimicrobial resistance, general stress response, and virulence. J. Bacteriol. 2011; 193(15):3740-3747. [PubMed: 21642465]

37. Jawad A, Seifert H, Snelling AM, Heritage J, Hawkey PM. Survival of Acinetobacter baumannii on dry surfaces: comparison of outbreak and sporadic isolates. J. Clin. Microbiol. 1998; 36(7): 1938-1941. [PubMed: 9650940]

38. Wendt C, Dietze B, Dietz E, Ruden H. Survival of Acinetobacter baumannii on dry surfaces. J. Clin. Microbiol. 1997; 35(6):1394-1397. [PubMed: 9163451]

39. Houang ET, Sormunen RT, Lai L, Chan CY, Leong AS. Effect of desiccation on the ultrastructural appearances of Acinetobacter baumannii and Acinetobacter lwoffii. J. Clin. Pathol. 1998; 51(10): 786-788. [PubMed: 10023344]

40. Espinal P, Marti S, Vila J. Effect of biofilm formation on the survival of Acinetobacter baumannii on dry surfaces. J. Hosp. Infect. 2012; 80(1):56-60. [PubMed: 21975219]

41. Villegas MV, Hartstein AI. Acinetobacter outbreaks, 1977-2000. Infect. Control Hosp. Epidemiol. 2003; 24(4):284-295. [PubMed: 12725359]

42. Mendez JA, Soares ND, Mateos JM, et al. Extracellular proteome of a highly invasive multidrugresistant clinical strain of Acinetobacter baumannii. J. Proteome Res. 2012; 11(12):5678-5694. [PubMed: 22966805]

43. Garnacho-Montero J, Amaya-Villar R. Multiresistant Acinetobacter baumannii infections: epidemiology and management. Curr. Opin. Infect. Dis. 2010; 23(4):332-339. [PubMed: 20581674]

44. Gordon NC, Wareham DW. Multidrug-resistant Acinetobacter baumannii mechanisms of virulence and resistance. Int. J. Antimicrob. Agents. 2010; 35(3):219-226. [PubMed: 20047818]

45. Siroy A, Molle V, Lemaitre-Guillier C, et al. Channel formation by CarO, the carbapenem resistance-associated outer membrane protein of Acinetobacter baumannii. Antimicrob. Agents Chemother. 2005; 49(12):4876-4883. [PubMed: 16304148]

46. Albina JE, Abate JA, Mastrofrancesco B. Role of ornithine as a proline precursor in healing wounds. J. Surg. Res. 1993; 55(1):97-102. [PubMed: 8105150]

47. Richet HM, Mohammed J, McDonald LC, Jarvis WR. Building communication networks: international network for the study and prevention of emerging antimicrobial resistance. Emerg. Infect. Dis. 2001; 7(2):319-322. [PubMed: 11294732] 
48. Poole K. Outer membranes and efflux: the path to multidrug resistance in Gramnegative bacteria. Curr. Pharm. Biotechnol. 2002; 3(2):77-98. [PubMed: 12022261]

49. Magnet S, Courvalin P, Lambert T. Resistance-nodulation-cell division-type efflux pump involved in aminoglycoside resistance in Acinetobacter baumannii strain BM4454. Antimicrob. Agents Chemother. 2001; 45(12):3375-3380. [PubMed: 11709311]

50. Coyne S, Rosenfeld N, Lambert T, Courvalin P, Perichon B. Overexpression of resistancenodulation- cell division pump AdeFGH confers multidrug resistance in Acinetobacter baumannii. Antimicrob. Agents Chemother. 2010; 54(10):4389-4393. [PubMed: 20696879]

51. Damier-Piolle L, Magnet S, Bremont S, Lambert T, Courvalin P. AdeIJK, a resistance-nodulationcell division pump effluxing multiple antibiotics in Acinetobacter baumannii. Antimicrob. Agents Chemother. 2008; 52(2):557-562. [PubMed: 18086852]

52. Vila J, Marti S, Sanchez-Cespedes J. Porins, efflux pumps and multidrug resistance in Acinetobacter baumannii. J. Antimicrob. Chemother. 2007; 59(6):1210-1215. [PubMed: 17324960]

53. Marchand I, Damier-Piolle L, Courvalin P, Lambert T. Expression of the RND-type efflux pump AdeABC in Acinetobacter baumannii is regulated by the AdeRS two-component system. Antimicrob. Agents Chemother. 2004; 48(9):3298-3304. [PubMed: 15328088]

54. Cardoso K, Gandra RF, Wisniewski ES, et al. DnaK and GroEL are induced in response to antibiotic and heat shock in Acinetobacter baumannii. J. Med. Microbiol. 2010; 59(Pt 9):10611068. [PubMed: 20576751]

55. Kohanski MA, Dwyer DJ, Hayete B, Lawrence CA, Collins JJ. A common mechanism of cellular death induced by bactericidal antibiotics. Cell. 2007; 130(5):797-810. [PubMed: 17803904] . Demonstrates that, in addition to working on specific targets, bactericidal antibiotics cause the generation of $\mathrm{OH}$ - radicals as a result of Fenton chemistry and damage to Fe-S clusters, resulting in bacterial death.

56. Sampson TR, Liu X, Schroeder MR, Kraft CS, Burd EM, Weiss DS. Rapid killing of Acinetobacter baumannii by polymyxins is mediated by a hydroxyl radical death pathway. Antimicrob. Agents Chemother. 2012; 56(11):5642-5649. [PubMed: 22908157]

57. Chen HP, Chen TL, Lai CH, et al. Predictors of mortality in Acinetobacter baumannii bacteremia. J. Microbiol. Immunol. Infect. 2005; 38(2):127-136. [PubMed: 15843858]

58. Chen MZ, Hsueh PR, Lee LN, Yu CJ, Yang PC, Luh KT. Severe community-acquired pneumonia due to Acinetobacter baumannii. Chest. 2001; 120(4):1072-1077. [PubMed: 11591541]

59. Kampf G, Kramer A. Epidemiologic background of hand hygiene and evaluation of the most important agents for scrubs and rubs. Clin. Microbiol. Rev. 2004; 17(4):863-893. [PubMed: 15489352]

60. Dixon B. There's the rub: infection control that spreads infection. Lancet Infect. Dis. 2008; 8(2): 91. [PubMed: 18222160]

61. Edwards J, Patel G, Wareham DW. Low concentrations of commercial alcohol hand rubs facilitate growth of and secretion of extracellular proteins by multidrug-resistant strains of Acinetobacter baumannii. J. Med. Microbiol. 2007; 56(Pt 12):1595-1599. [PubMed: 18033825]

62. Smith MG, Des Etages SG, Snyder M. Microbial synergy via an ethanol-triggered pathway. Mol. Cell Biol. 2004; 24(9):3874-3884. [PubMed: 15082781]

63. Camarena L, Bruno V, Euskirchen G, Poggio S, Snyder M. Molecular mechanisms of ethanolinduced pathogenesis revealed by RNA-sequencing. PLoS Pathog. 2010; 6(4):e1000834. [PubMed: 20368969] . - Transcriptomic analysis of $A$. baumannii ATCC 17978 grown in the presence of ethanol that shows the upregulation of genes associated with ethanol assimilation and stress-protection processes.

64. Nwugo CC, Arivett BA, Zimbler DL, Gaddy JA, Richards AM, Actis LA. Effect of ethanol on differential protein production and expression of potential virulence functions in the opportunistic pathogen Acinetobacter baumannii. PLoS One. 2012; 7(12):e51936. [PubMed: 23284824]

65. Flemming HC, Wingender J. The biofilm matrix. Nat. Rev. Microbiol. 2010; 8(9):623-633. [PubMed: 20676145]

66. Verstraeten N, Braeken K, Debkumari B, et al. Living on a surface. swarming and biofilm formation. Trends Microbiol. 2008; 16(10):496-506. [PubMed: 18775660] 
67. Bianco C, Imperlini E, Calogero R, et al. Indole-3-acetic acid improves Escherichia coli's defences to stress. Arch. Microbiol. 2006; 185(5):373-382. [PubMed: 16555073]

68. Bianco C, Imperlini E, Calogero R, Senatore B, Pucci P, Defez R. Indole-3-acetic acid regulates the central metabolic pathways in Escherichia coli. Microbiology. 2006; 152(Pt 8):2421-2431. [PubMed: 16849805]

69. Hoshi A, Oshima K, Kakizawa S, et al. A unique virulence factor for proliferation and dwarfism in plants identified from a phytopathogenic bacterium. Proc. Natl Acad. Sci. USA. 2009; 106(15): 6416-6421. [PubMed: 19329488]

70. Yang S, Zhang Q, Guo J, et al. Global effect of indole-3-acetic acid biosynthesis on multiple virulence factors of Erwinia chrysanthemi 3937. Appl. Environ. Microbiol. 2007; 73(4):1079_ 1088. [PubMed: 17189441]

71. Smith MG, Gianoulis TA, Pukatzki S, et al. New insights into Acinetobacter baumannii pathogenesis revealed by high-density pyrosequencing and transposon mutagenesis. Genes Dev. 2007; 21(5):601-614. [PubMed: 17344419]

72. Spaepen S, Vanderleyden J, Remans R. Indole-3-acetic acid in microbial and microorganism-plant signaling. FEMS Microbiol. Rev. 2007; 31(4):425-448. [PubMed: 17509086]

73. Lin GH, Chen HP, Huang JH, et al. Identification and characterization of an indigo-producing oxygenase involved in indole 3-acetic acid utilization by Acinetobacter baumannii. Antonie Van Leeuwenhoek. 2012; 101(4):881-890. [PubMed: 22311185]

74. Chilcote DD. Column-chromatographic analysis of naturally fluorescing compounds. II. Rapid analysis of indoleacetic acid and 5-hydroxyindoleacetic acid in biological samples. Clin. Chem. 1972; 18(11):1376-1378. [PubMed: 4652836]

75. Morita I, Kawamoto M, Hattori M, Eguchi K, Sekiba K, Yoshida H. Determination of tryptophan and its metabolites in human plasma and serum by high-performance liquid chromatography with automated sample clean-up system. J. Chromatogr. 1990; 526(2):367-374. [PubMed: 2361979]

76. Young SN, Gauthier S. Effect of tryptophan administration on tryptophan, 5-hydroxyindoleacetic acid and indoleacetic acid in human lumbar and cisternal cerebrospinal fluid. J. Neurol.

Neurosurg. Psychiatry. 1981; 44(4):323-328. [PubMed: 6165809] 


\section{Executive summary}

\section{Background}

- Acinetobacter baumannii persists under harsh conditions in the medical environment and on devices used in human medicine.

- A. baumannii colonizes and causes severe human infections by evading the effects of host defense factors and antimicrobials.

- Understanding the action and effects of stressors encountered by $A$. baumannii during environmental persistence and host colonization/infection will facilitate the comprehension of its pathobiology.

\section{Effects \& responses to environmental \& cellular stressors}

- Limitation of essential nutrients, such as iron, induces a specific response aimed at acquiring these nutrients, as well as a general stress response that facilitates the persistence of $A$. baumannii in medical settings and its interaction with the human host.

- Bacterial growth and the presence of antibiotics cause $A$. baumannii to mount complex stress responses that protect it from a wide range of cellular and molecular damage caused by reactive metabolic products and enhance resistance of this pathogen to antibiotics and host effectors.

- Presence of antiseptics, such as ethanol, could favor the persistence and enhance the virulence of $A$. baumannii by serving as a convenient nutrient, as well as inducing a global response that protects bacteria from stress and promotes important host-pathogen interactions.

\section{Future perspective}

- Multi-/inter-disciplinary approaches combined with genome-wide studies and standard virulence/pathogenesis methods should provide a comprehensive understanding of the nature of molecular and cellular mechanisms, as well as their interaction, which are responsible for the virulence of $A$. baumannii.

- Understanding the pathobiology of $A$. baumannii could facilitate the development of alternative therapeutics needed for the treatment of infections caused by multidrug-resistant strains. 

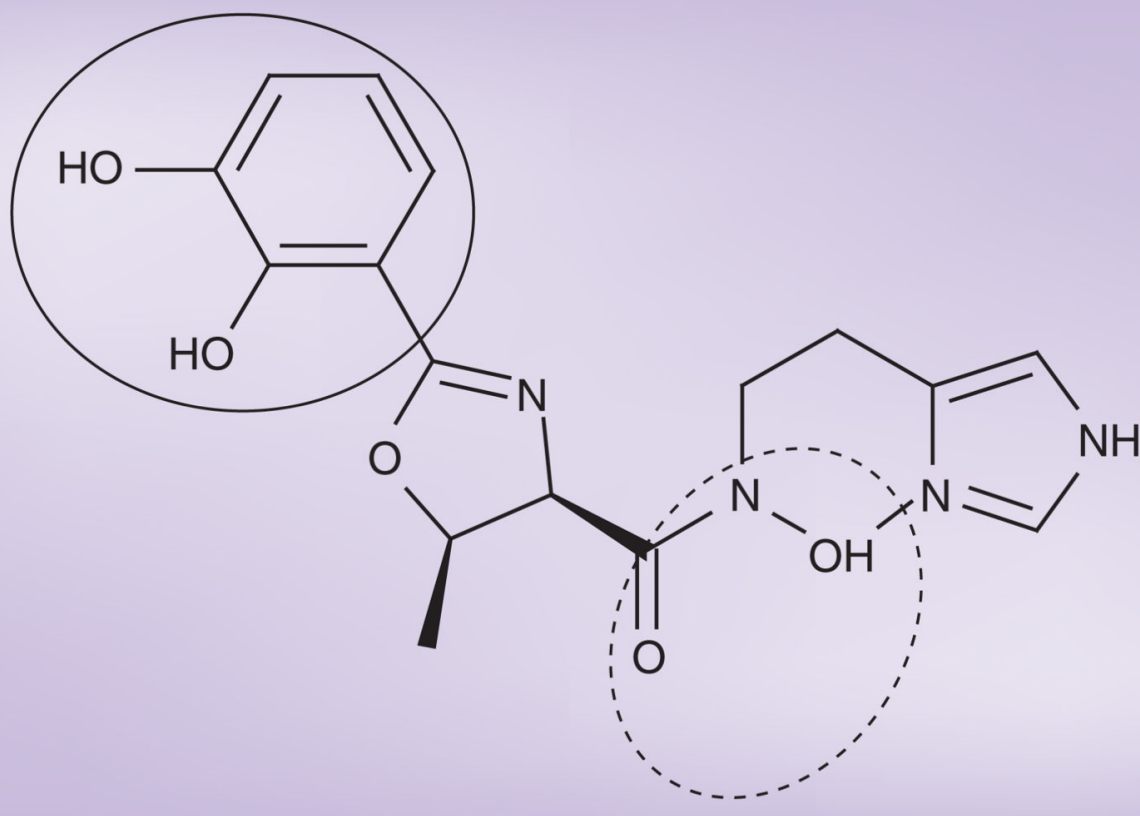

Figure 1. Structure of the Acinetobacter baumannii ATCC $19606^{\mathrm{T}}$ siderophore acinetobactin The catechol and hydroxamate moieties are shown within the solid and dashed ovals, respectively. 


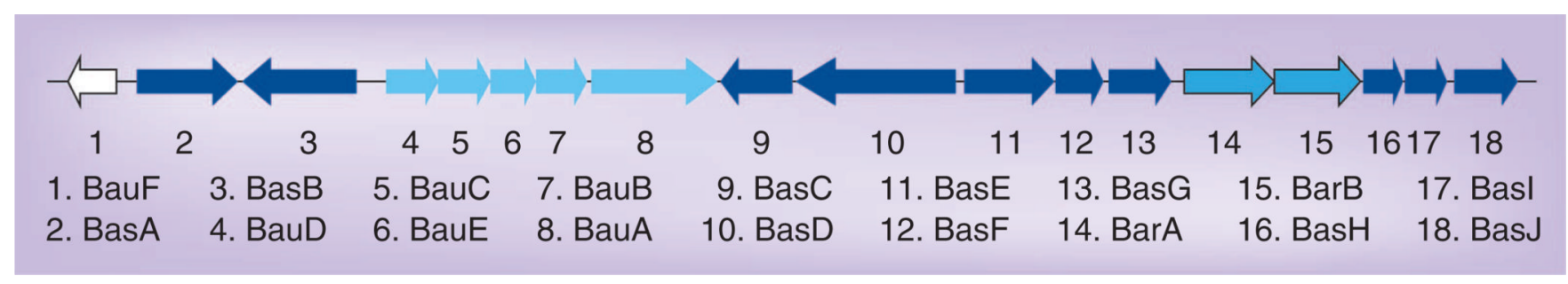

Figure 2. Genetic structure of the Acinetobacter baumannii ATCC $19606^{\mathrm{T}}$ acinetobactin locus Gene functions are depicted as follows: dark block arrows, acinetobactin biosynthesis; light block arrows, ferric acinetobactin import; light outlined arrows, acinetobactin export; white outlined arrow, acinetobactin degradation. 


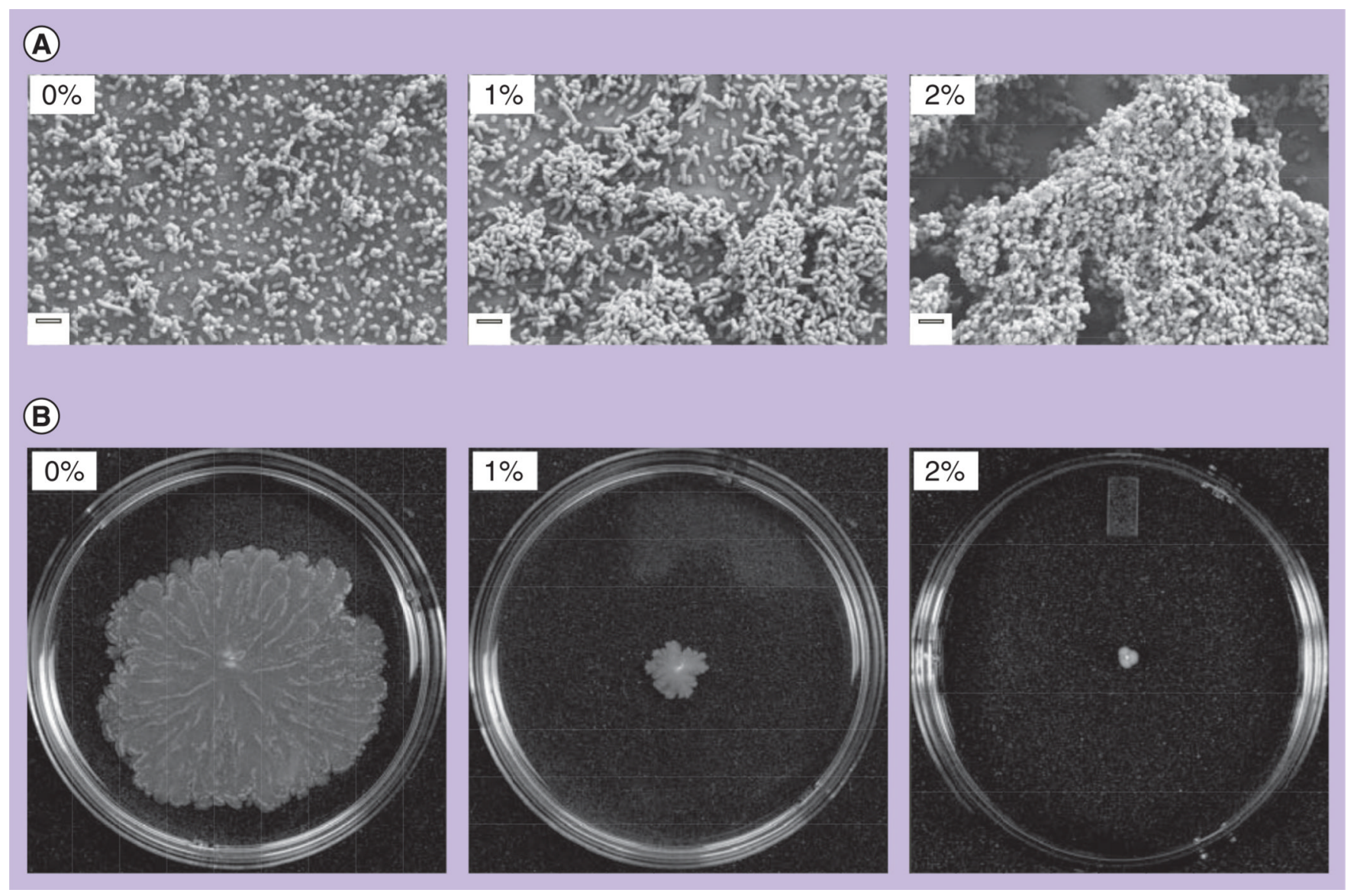

Figure 3. Effect of ethanol on Acinetobacter baumannii ATCC 17978 biofilm formation and surface motility

(A) Biofilms formed by ATCC 17978 cells on plastic coverslips at the liquid-air interface after overnight incubation at $37^{\circ} \mathrm{C}$ in Luria-Bertani broth either containing no ethanol $(0 \%)$ or supplemented with 1 or $2 \%$ ethanol were processed and visualized by scanning electron microscopy. (B) Motility of ATCC 17978 cells was tested using $0.3 \%$ agarose plates containing no ethanol $(0 \%)$ or supplemented with 1 or $2 \%$ ethanol. Results were recorded after overnight incubation at $37^{\circ} \mathrm{C}$.

Scale bars: $2 \mu \mathrm{m}$. 